\title{
PREPARATION OF THE SINGLE MODE PLANAR OPTICAL SPLITTER MODULES AND THEIR CHARACTERIZATIONS
}

\author{
Vu Doan Mien* \\ Institute of Materials Science, \\ Vietnamese Academy of Science and Technology, \\ 18, Hoang Quoc Viet Rd., Cau Giay, Hanoi, Vietnam \\ College of Technology, National University of Vietnam, \\ 144 Xuan Thuy, Cau Giay, Hanoi, Vietnam \\ Vu Thi Nghiem and Pham Van Truong \\ Institute of Materials Science, \\ Vietnamese Academy of Science and Technology, \\ 18, Hoang Quoc Viet Rd., Cau Giay, Hanoi, Vietnam
}

Received 27 November 2006

\begin{abstract}
Optical splitter modules have been prepared based on $1 \times 8$ single mode silica planar waveguide optical splitter chips with $250 \mu \mathrm{m}$ spacing and v-groove fiber arrays for applications in fiber optic communications. We report the technology of precise optical coupling and packaging of the splitter modules and the measurements of the insertion loss $(<11 \mathrm{~dB})$, uniformity $(<0.80$ $\mathrm{dB})$ and polarization dependence loss (PLD $<0.10 \mathrm{~dB})$ as well as the lateral profile and the image of the input and output lights for the wavelengths of $1310 \mathrm{~nm}$ and $1550 \mathrm{~nm}$. The main characteristics of the prepared splitter modules are about the same for the commercial available products. The prepared modules have been tested for operation in the conditions of wide temperature range $\left(5-80^{\circ} \mathrm{C}\right)$ and humidity range $(50-98 \%)$ and no changes in the main characteristics were observed.
\end{abstract}

Keywords: Optical planar waveguide splitter module, v-groove fiber, optical coupling and packaging, insertion loss, PLD

\section{INTRODUCTION}

Optical planar waveguides are the key element in integrated optics [1]. They consist of a region with high refractive index surrounded with regions of lower refractive index. The optical splitters are one kind of the passive optical waveguide devices which are used in fiber optic communications for dividing the optical power of the signals or combining them [2]. Especially, they are widely used in the cable television systems (CATV) where the optical fiber is used as transmission medium. In comparison with the fiber splitter, the planar waveguide splitters have

\footnotetext{
* Corresponding author e-mail: mienvd@ims.vast.ac.vn
} 
the advantages in compactness, better uniformity when the number of output ports is large $(8,16$, etc. and ability to integrate with the other photonic components to form the optoelectronics integrated circuits. The inorganic planar splitters have a low insertion loss, low polarization dependence loss (PDL) while polymer-based planar splitters are potentially less costly and versatile [3]. In this paper we report the preparation of $1 \times 8$ single mode silica optical planar waveguide splitter modules based on the inorganic SOI (Silica-On-Isolator) planar splitter chips and v-groove fiber arrays. We also present the measured results of the main characteristics for the prepared planar splitter modules and some measurement results for polymer planar waveguide splitter chips for comparison.

\section{EXPERIMENTS RESULTS AND DISCUSSIONS}

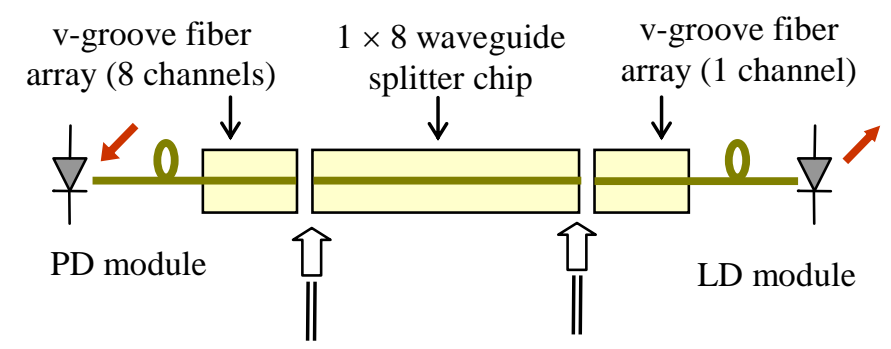

$\begin{array}{lc}\text { White light } & \text { White light } \\ \text { LED module } & \text { LED module }\end{array}$

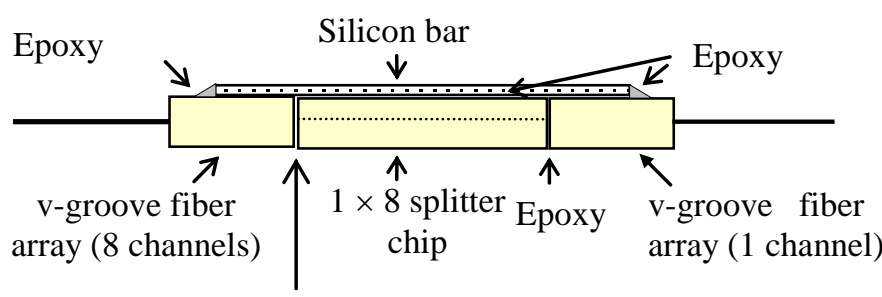

b) Slit $\sim 1-2 \mu \mathrm{m}$

Fig. 1: Alignment (a) and fixing (b) processes of the optical waveguide splitter module

The silica optical planar waveguide splitter chips (SOI) from Wooriro company and its fiber arrays in v-groove form were used for making planar optical splitters. The fiber arrays have the FC connectors at their ends. The splitter chips and fiber array have the sandwich structure where the waveguides and 9/125 single mode fibers are placed between two quartz substrates. Splitter chips and fiber arrays have the angled-facet polishing sides with the angle of $8^{\circ}$ for decreasing the reflections from the interface of silica and air. The splitter chips have the dimensions of $2.5 \times 2.5 \times 10 \mathrm{~mm}$ and the spacing between waveguides is $250 \mu \mathrm{m}$. The waveguide channels have the cross-section dimensions of $5 \times 5 \mu \mathrm{m}^{2}$. To make optical coupling we use the specialized for waveguide coupling six-axis Konzu Fine Pitch Positioner FPP03-13 with sub-micron positioning accuracy and high magnification stereomicroscope AMS-JENA. The single mode InGaAsP/InP FP and DFB laser modules $(\lambda=1310 \mathrm{~nm}$ and $1550 \mathrm{~nm})$ which were prepared by us were used as the optical signal sources for alignment and characterization of the prepared splitter modules. The output light signals were detected with PIN InGaAs photodiode modules which were prepared also by us. The process of coupling was difficult 
because we needed to have the exact alignment at the same time for both input port ( 1 channel) and output ports ( 8 channels) of the splitter chips with the fiber arrays. The best results were achieved when the distance between splitter chip and fiber array is around 1 or $2 \mu \mathrm{m}$. To make the transparent waveguide channels to become visible we used the white light LED modules that were prepared by us based on the white light LED and plastic optical fiber. After the alignment we use the small silicon bar served as a lid to connect the waveguide chip and v-groove fiber arrays. After all we fixed splitter chip and the input and output fiber arrays using epoxy Araldite 2014 and put them into the metallic cover which was hermetically sealed with the epoxy. The hardening of epoxy was performed at the temperature of $60^{\circ} \mathrm{C}$ during several hours. Figure 1 shows the alignment (a) and fixing (b) processes for optical waveguide chip and vgroove fiber arrays. After packaging, the splitter modules were thermally treated in the temperature case at the temperature of $70^{\circ} \mathrm{C}$ during 8 hours for the stabilization in operation. The packaging technique was developed from our experiences in optical module packaging [1]. Figure 2 shows the image of the prepared splitter module.

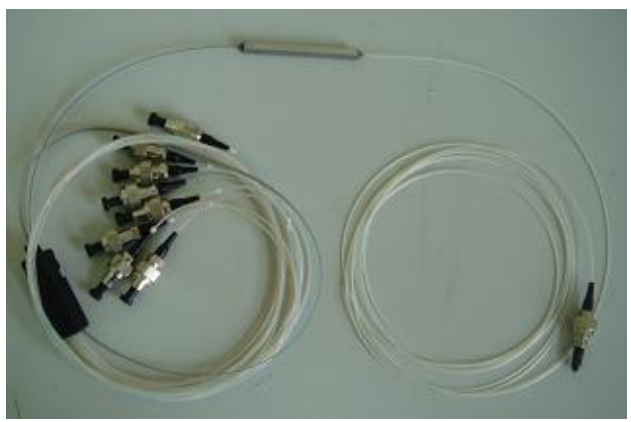

Fig. 2: The image of the prepared $1 \times 8$ optical waveguide splitter module

Figure 3 presents the experimental setup for measurement of the main characteristics of the splitter modules. The optical power meter KI 7600 GE (Kingfisher) with the sensitivity of \pm 1 $\mathrm{nW}$ was used. The $1310 \mathrm{~nm}$ and $1550 \mathrm{~nm} \mathrm{LD}$ modules were put on the peltier elements for stabilizing their output power. The prepared splitter modules were measured for the total coupling efficiency $\eta\left(\eta=\Sigma \mathrm{P}_{\text {out,i }} / \mathrm{P}_{\text {in }}\right.$, where $\mathrm{P}_{\text {out,i }}$ is the output power of the $i$ output channel, $\mathrm{P}_{\text {in }}$ is the input power) which is equal the sum of coupling efficiency and splitter chip waveguide efficiency. The insertion $\operatorname{loss}\left(-10 \log \left(\mathrm{P}_{\text {out }, i} / \mathrm{P}_{\text {in }}\right)\right)$ was measured for each output channel with the accuracy of $\pm 0.10 \mathrm{~dB}$. The uniformity of the output channel power distribution and the polarization dependence loss (PDL) were also measured. Table 1 presents the measurement results of the main characteristics of four prepared $1 \times 8$ splitter modules and for comparison we also present the measured properties of two $1 \times 8$ commercial splitter modules of the company TeemPhotonics (France). Our prepared modules have the total coupling efficiency $(\eta=65-$ $70 \%$ ) of more than $90 \%$ in comparison with the commercial ones so the average insertion loss $(<11 \mathrm{~dB})$ is a little bit more. The uniformity $(<0.80 \mathrm{~dB})$ and PLD $(<0.10 \mathrm{~dB})$ of our prepared modules are the same as for the commercial ones. Figure 4 shows the insertion loss curves for the output channels of the prepared $1 \times 8$ optical splitter modules at $1550 \mathrm{~nm}$.

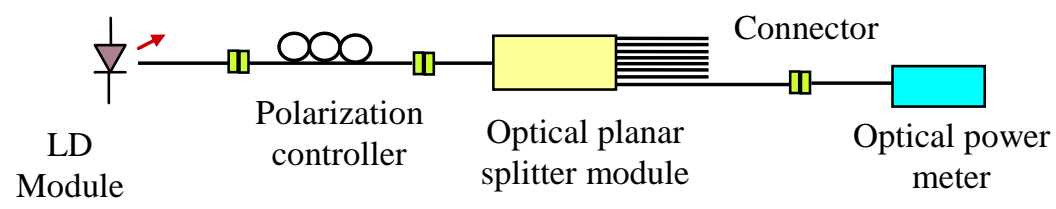

Fig. 3: Experimental setup for splitter module characterizations 
Table 1: Main characteristics of the optical planar waveguide splitter modules

\begin{tabular}{lccccccc}
\hline $\begin{array}{l}1 \times 8 \text { optical } \\
\text { splitter module }\end{array}$ & \multicolumn{2}{c}{$\begin{array}{c}\text { Total coupling } \\
\text { efficiency } \eta, \%\end{array}$} & \multicolumn{2}{c}{$\begin{array}{c}\text { Average insertion } \\
\text { loss, dB }\end{array}$} & Uniformity, dB & PLD, dB \\
\cline { 2 - 8 } & $1310 \mathrm{~nm}$ & $1550 \mathrm{~nm}$ & $1310 \mathrm{~nm}$ & $1550 \mathrm{~nm}$ & $1310 \mathrm{~nm}$ & $1550 \mathrm{~nm}$ & $1550 \mathrm{~nm}$ \\
\hline Prepared N1 & 66.8 & 69.5 & 10.80 & 10.62 & 0.45 & 0.56 & 0.09 \\
Prepared N2 & 71.0 & 67.5 & 10.54 & 10.74 & 0.35 & 0.18 & 0.09 \\
Prepared N3 & 64.9 & 67.9 & 10.93 & 10.72 & 0.54 & 0.47 & 0.08 \\
Prepared N4 & 64.4 & 65.5 & 10.96 & 10.86 & 0.50 & 0.45 & 0.09 \\
$\begin{array}{l}\text { Commercial N1 } \\
\text { (TeemPhoton.) }\end{array}$ & 72.3 & 72.1 & 10.46 & 10.47 & 0.78 & 0.79 & 0.08 \\
$\begin{array}{l}\text { Commercial N2 } \\
\text { (TeemPhoton.) }\end{array}$ & 76.2 & 75.3 & 10.22 & 10.26 & 0.40 & 0.38 & 0.08 \\
\hline
\end{tabular}

When the splitter modules work as a combiner to combine the different light signal powers it is very important that the signal from one channel does not influence the signal from another one. For our prepared splitter modules this kind of crosstalk is very low $(<-50 \mathrm{~dB})$ while for the commercial (TeemPhotonics Com.) splitter modules the crosstalk is much more higher $(<-30 \mathrm{~dB})$.

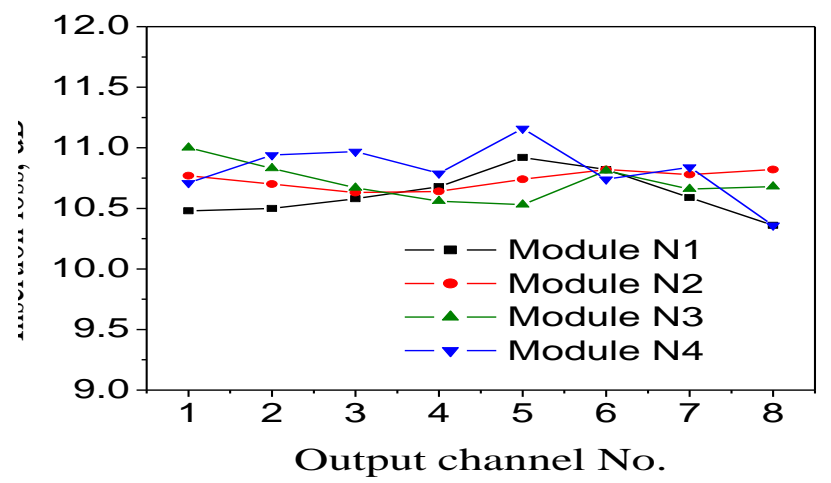

Fig. 4: Insertion loss of the output channels of the prepared $1 \times 8$ optical splitter modules at $1550 \mathrm{~nm}$

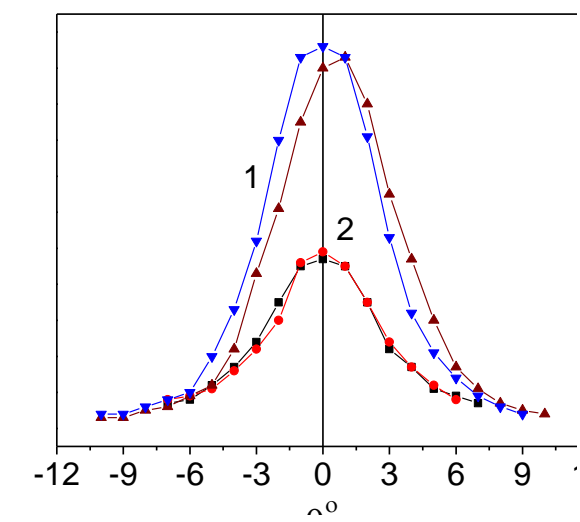

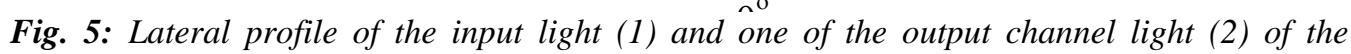
prepared module $\mathrm{N} 4$ 
The lateral profile and the image of the input and output lights for the wavelengths of $1310 \mathrm{~nm}$ and $1550 \mathrm{~nm}$ also were measured. The lateral profile (Fig. 5) and the images (Fig. 6) are the same for the input and output channels of the splitters modules. The lateral profiles for both perpendicular direction have the gauss distribution and are the same for all the output channels. The input and output light images were measured by using infrared viewing card and the last are the same for all the output channels. We also measured the optical spectra of the input and output channel light of the modules and found that the spectra in general have no change in their shape.

For comparison we carried out the measurements for the $1 \times 4$ polymer-based optical planar splitter chips which were made in Heindrich Hertz Institute (Berlin, Germany). They have a lower total coupling efficiency $(\eta \sim 40 \%)$, higher insertion loss and it depends very much on the input light polarization $(\mathrm{PDL} \leq 2 \mathrm{~dB})[5]$.

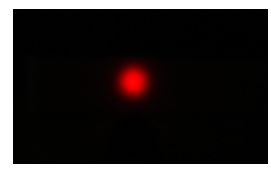

a)

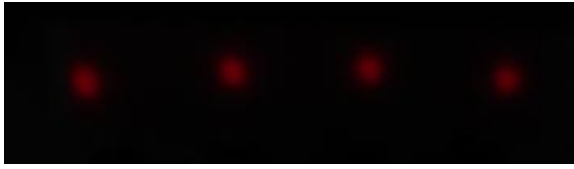

1

2

3

4

b)

Fig. 6: Image of the input light (a) and output channel lights $(b)$ of the prepared splitter module N4

We performed the treatment for the prepared optical planar splitter modules in the conditions of high and low temperatures by putting them in the temperature cases with temperature range changing from $5^{\circ} \mathrm{C}$ to $80^{\circ} \mathrm{C}$ (using peltier cooler and furnace) and high humidity (50\% to $98 \%$ ). Figure 7 presents the insertion loss curves for the output channels of the prepared module N1. No change in the main splitter characteristics of all the prepared modules was observed. The module characteristics also are stabilized during more than one year operation in CATV systems.

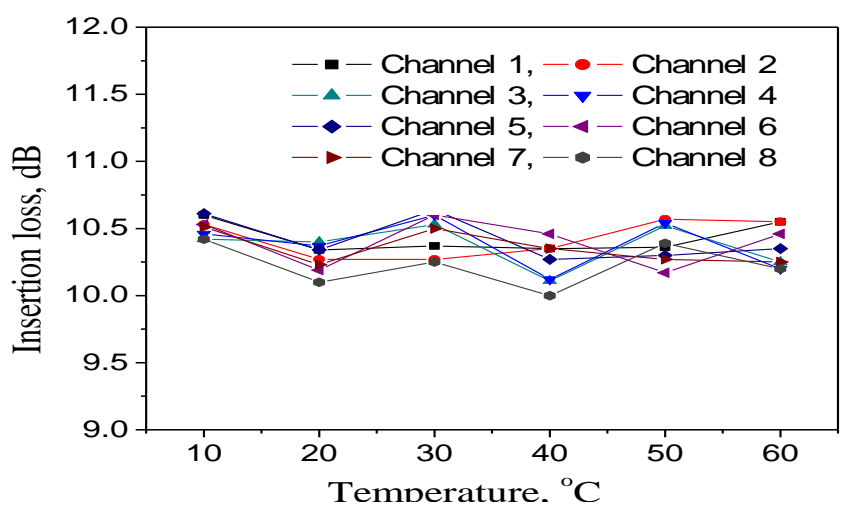

Fig. 7: Dependence of insertion loss on temperature for the different output channels of the prepared module $\mathrm{NI}$ 


\section{CONCLUSION}

For the first time in Vietnam we have succeeded in packaging the $1 \times 8$ optical planar splitter modules based on the silica planar splitter chips and v-groove fiber arrays with the characteristics comparable with the commercial available planar splitters. The prepared splitter modules were tested in the aging conditions and can be used in the fiber optic communication systems and cable television systems for power splitting of the optical signals.

\section{ACKNOWLEDGEMENT}

The authors express sincere thanks to the Vietnamese Academy of Science and Technology for its financial supporting and Prof. Acad. Nguyen Van Hieu for his permanent support for this work.

\section{REFERENCES}

1. Roberg G. Hunsperger (2002), Integrated Optics: Theory and Technology. Fifth Edition. Springer. Verlag, Berlin, Heidenberg, New York.

2. Hyun - Chae Song, Tae-Won Oh, Sang-Yung Shin, Member, IEEE, Sang-Yun Yi, WooHyuk Jang, and Tae Hyung Rhee (1998), Four- Brach Single- Mode Waveguide Power Divider, IEEE Photonic Techonogy Letters, vol.10, No.129, pp. 1760-1762.

3. Hong Ma, Alex K.Y. Jen, and Larry R. Dalton (2002), Polymer-Based Optical Waveguides: Materials, Processing, and Devices. Advanced Materials, vol. 14(19), pp. 1339-1365.

4. Vu Van Luc, Vu Doan Mien, Luong Vu Hai Nam, Pham Van Truong, and Vu Thi Nghiem (2005), Advances in Photonics and Applications. Vietnam National University Publisher, pp. 141-145 (Proceeding of the International Workshop on Photonics and ApplicationIWPA, 5 - 8 April 2004, Hanoi, Vietnam).

5. Vu Doan Mien, Le Quoc Minh, Vu Thi Nghiem, Phan Viet Phong, and Luong Vu Hai Nam (2005), Some first results of the study of $1 \times 4$ and $1 \times 8$ optical planar wave guide splitter chips. Proceedings of the 6th National Physics Conference, Hanoi, 23 - 25 November, 2005, vol. 1, pp. 390-393. 